\title{
Pervasive artifacts revealed from magnetometry measurements of rare earth-transition metal thin films
}

\author{
Cite as: J. Vac. Sci. Technol. A 38, 023409 (2020); doi: 10.11 16/1.5135504 \\ Submitted: 6 November 2019 - Accepted: 10 February 2020 . \\ Published Online: 4 March 2020
}

Andrada-Oana Mandru, ${ }^{7, a)}$ (D) Oğuz Yıldırım, ${ }^{1}$ Miguel A. Marioni, ${ }^{1, b)}$ Hartmut Rohrmann, ${ }^{2}$ Michael Heigl, ${ }^{3}$ Oana-Tereza Ciubotariu, ${ }^{3}$ Marcos Penedo, ${ }^{7}$ Xue Zhao, ${ }^{2}$ Manfred Albrecht, ${ }^{3}$ and Hans J. Hug ${ }^{1,4}$

\author{
AFFILIATIONS \\ ${ }^{1}$ Empa, Swiss Federal Laboratories for Materials Science and Technology, $\mathrm{CH}-8600$ Dübendorf, Switzerland \\ ${ }^{2}$ Evatec AG, CH-9477 Trübbach, Switzerland \\ ${ }^{3}$ Institute of Physics, University of Augsburg, D-86135 Augsburg, Germany \\ ${ }^{4}$ Department of Physics, University of Basel, $\mathrm{CH}-4056$ Basel, Switzerland
}

Note: This paper is part of the Special Topic Collection on 30 years of the Nellie Yeoh Whetten Award - Celebrating the Women of the AVS.

${ }^{a}$ Electronic mail: andrada-oana.mandru@empa.ch

b) Deceased.

\begin{abstract}
A class of artifacts manifesting as soft magnetic components are revealed from magnetometry measurements of rare earth-transition metal $(\mathrm{TbFe})$ thin films prepared by magnetron sputtering. They are not inherent to TbFe, but are a direct result of the manner in which the substrates are mounted prior to sample fabrication, with a material deposited at the substrate sides giving rise to a significant magnetic moment. The authors find the same artifacts to also be present in rare earth-free [Co/Pt] multilayers. Trying to supress the appearance of this type of artifact has an influence on the coercivity and, in some cases, on the shape of the reversal curves. Care needs to be taken during fabrication to ensure reliable and reproducible samples so that sensitive magnetic parameters, such as coercivity and compensation points, can be extracted accurately and that data are not misinterpreted for even more complex systems. This type of artifact is not limited to samples prepared by sputtering but can extend to other conventional thin-film deposition methods.
\end{abstract}

Published under license by AVS. https://doi.org/10.1116/1.5135504

\section{INTRODUCTION}

Amorphous rare earth-transition metal (RE-TM) alloys exhibit a rich spectrum of magnetic properties. They are ferrimagnetic (FI) with compensation points that cover a large temperature range, can exhibit perpendicular magnetic anisotropy (PMA), and can have very high coercive fields for certain compositions. ${ }^{1-5}$ These properties make them attractive pinning layers as an alternative to conventional antiferromagnets such that when coupled with perpendicular FI or ferromagnetic (FM) layers, they can be exploited in exchangebias systems and heat-assisted magnetic recording applications. ${ }^{4,6-14}$ More recently, RE-TM alloys have been investigated in the vicinity of their magnetization and angular momentum compensation points for high-speed domain wall- and skyrmion racetrack-type memory devices. ${ }^{15-20}$ The high magnetic tunability of RE-TM ferrimagnet-based heterostructures allows them to be tailored to specific applications, provided that their magnetic parameters are determined accurately. For the specific case of $\mathrm{Tb}(\mathrm{Co}) \mathrm{Fe}$, which is known to be very sensitive to the growth conditions, ${ }^{2,21-23}$ the existence of soft magnetic characteristics in the hysteresis loops of an otherwise magnetically hard film has been observed and mainly explained as the result of preferential oxidation of the RE element or chemical inhomogeneities. ${ }^{23-26}$ However, in such complex systems, distinguishing between real magnetic features and artifacts can be challenging and requires some caution.

Volume magnetometry tools, such as superconducting quantum interference device (SQUID) and vibrating sample 
magnetometry (VSM), are primarily used to determine the magnetic properties of the above mentioned systems. Although well established, magnetometry measurements are not error-free and care needs to be taken during measurements to ensure reliable and reproducible results. For instance, there have been reports of various issues that can occur during magnetometry measurements, such as inadequate sample positioning and handling, sample holder off-centering, contaminations/impurities, etc., that make an interpretation of the data not trivial. ${ }^{27-39}$ Even though the VSM and SQUID systems exist with best-practice protocols, these precautions do not address other potential problems, such as those arising at the level of sample fabrication and preparation.

In this study, we reveal a class of artifacts manifesting as soft magnetic components in the hysteresis loops of various composition TbFe alloys. We show that these artifacts are due to the manner in which the substrates are mounted during sputter depositions. We further show that the observed effects can also impact systems that do not contain RE elements, such as $[\mathrm{Co} / \mathrm{Pt}]$ multilayers. As numerous studies investigate exchange-coupling in $\mathrm{TbFe} /$ $[\mathrm{Co} / \mathrm{Pt}]$ or similar systems, the issues that we describe here become exceedingly important when interpreting the coupling and magnetization reversal processes in such heterostructures. Moreover, we argue that these observations can also be relevant for magnetic thin-film systems prepared by methods other than sputtering.

\section{EXPERIMENT}

Magnetometry measurements generally require that the sample dimensions are relatively small $\left(5 \times 5 \mathrm{~mm}^{2}\right)$ and such samples are often obtained by cutting after depositing on a larger substrate. In some respects this procedure can be inconvenient. For example, particle contamination may not be avoided during the cutting process; even if the contamination is not magnetic, it can become extremely problematic in surface studies that complement the magnetometry measurements, particularly in non-contact magnetic force microscopy where the tip-sample distance is kept at $\approx 10 \mathrm{~nm}$ during scanning. ${ }^{40,41}$ In addition, magnetic material from the cutting tools can in some cases contaminate the samples, leading to false ferromagnetic signals causing serious issues for small magnetic moment samples. Another example is the case of highly magnetostrictive systems, where the potential mechanical deformation resulting from cutting constitutes an additional source of uncertainty. Due to these reasons, it is convenient to use precut substrates rather than large wafers that would require post-deposition cutting.

Naturally oxidized $\mathrm{Si}(100)$ substrates with dimensions of $2.2 \times 2.2$ and $6 \times 6 \mathrm{~mm}^{2}$ are used for the films in this study, with selected samples also grown on thermally oxidized $\mathrm{Si}(100)$ for comparison. We prepare three different thin-film sample systems using DC magnetron sputtering in an AJA Orion system with base pressure of $\approx 1 \times 10^{-9}$ mbar: (i) $20 \mathrm{~nm}$-thick $\mathrm{Tb}_{1-x} \mathrm{Fe}_{x}$ films (with nominal $x=65,70,75$, and 85 at.\%), (ii) $\left[\mathrm{Co}_{0.4 \mathrm{~nm}} / \mathrm{Pt}_{0.7 \mathrm{~nm}}\right]_{\times 5} \mathrm{mul}-$ tilayers, and (iii) $\mathrm{Tb}_{1-x} \mathrm{Fe}_{x}(20 \mathrm{~nm}) /\left[\mathrm{Co}_{0.4 \mathrm{~nm}} / \mathrm{Pt}_{0.7 \mathrm{~nm}}\right]_{\times 5}$ coupled layers (with $x$ the same as above). Prior to deposition, the Si substrates are annealed in situ at $\approx 100^{\circ} \mathrm{C}$ for $1 \mathrm{~h}$. All depositions are performed at room temperature on Pt-seed layers, and all samples are capped with Pt to prevent oxidation. The Ar pressure during sputtering is $2 \mu$ bar for all sample systems. The TbFe films are prepared by co-deposition sputtering; in order to obtain differentcomposition films, the $\mathrm{Tb}$ target power is kept constant while the Fe target power is changed between the four samples. The magnetic properties are investigated using a Quantum Design VSM that allows up to $70 \mathrm{kOe}$ magnetic fields to be applied over a $1.9-400 \mathrm{~K}$ temperature range. Due to the fact that all investigated systems exhibit PMA, the VSM measurements are performed primarily in the out-of-plane geometry. Additional magneto-optic Kerr effect (MOKE) measurements are carried out at room temperature on the $6 \times 6 \mathrm{~mm}^{2}$ samples using a home-built setup in the polar geometry with a magnetic field range of $\pm 10 \mathrm{kOe}$.

\section{RESULTS AND DISCUSSION}

Shown in Fig. 1(a) is a picture of the substrate holder used during the sputtering process. The smaller-size substrates are fixed to the holder in two different configurations: no mask-one of the more typical mounting schemes during sputter depositions, in which the substrates are glued to the holder using silver paint and are fully exposed (whole top surface and sides) to the incoming flux and mask-substrates are placed inside tight, precut spaces of similar size within the holder, and then covered by a circular mask that leaves a central fraction of the top surface exposed. In spite of the aforementioned issues that can arise, sample cutting is a common practice in the magnetometry community. Therefore, for comparison purpose, we also mount larger $6 \times 6 \mathrm{~mm}^{2}$ substrates (similarly to the no mask case above) to subsequently cut smaller pieces. The cutting procedure was performed as follows: the sample was placed with the coated part facing down on a lint-free lab wiper and four scratches delimiting an area similar to the one of the small-size substrate were made using a diamond pen. Then, using plastic tweezers this excess material was snapped off; the resulting sample represents a third configuration, i.e., cut.

The VSM background signal coming from the out-of-plane holder together with a Si substrate is shown in Fig. 1(b) for the entire available field range and for two temperatures, 300 and $100 \mathrm{~K}$. The inset emphasizes the absence of any ferromagnetic signal in the low field regime. This paramagnetic background was subtracted from all loops. In order to minimize errors, all samples were measured using the same VSM holder. Furthermore, the background signal was periodically remeasured and was reproduced within less than $10 \%$. Note that since silver paint was used to fix the no mask samples, we also remeasured the background for a $\mathrm{Si}$ substrate after glueing, annealing, and allowing it to cool down to room temperature. The results (not shown) still do not indicate the presence of a ferromagnetic signal and therefore any contamination coming from the paint can be excluded.

The VSM loops for a $\mathrm{Tb}_{30} \mathrm{Fe}_{70}$ film recorded at $300 \mathrm{~K}$ for the three sample configurations described above are presented in Figs. 2(a), 2(b), and 2(d)-2(f). Considering the no mask and mask cases from Figs. 2(a) and 2(b), where the loops are displayed over the entire field range $(-70$ to $+70 \mathrm{kOe})$, two observations can be made. One is the further increase in magnetization with respect to the remnant value (see green ellipses); this is a known effect arising from the sperimagnetic nature of the (Tb-rich) TbFe alloys ${ }^{42}$ and was also observed in samples grown by other groups. ${ }^{3}$ The 

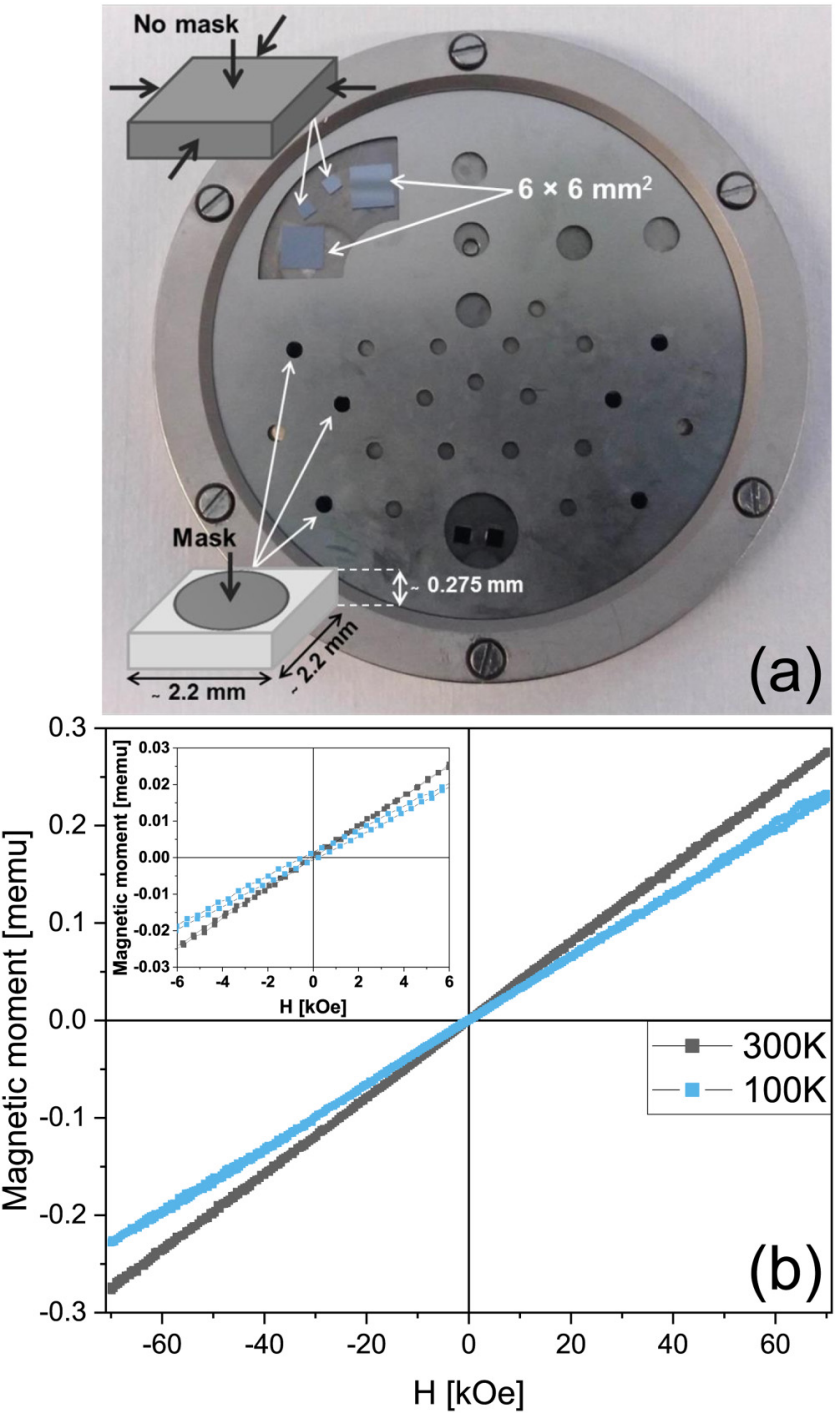

FIG. 1. (a) Picture of the sample holder used during the sputtering process together with schematics of two different sample configurations: no mask-in which the entire substrate is exposed to the incoming flux and mask-in which a circular mask allows for only a fraction of the top surface to be exposed; also visible are two larger substrates $\left(6 \times 6 \mathrm{~mm}^{2}\right)$ from where smaller-size samples were cut. (b) Background measurement (including a $\mathrm{Si}$ substrate) at 300 and $100 \mathrm{~K}$ for the out-of-plane holder used in all VSM measurements; the inset shows a zoom-in to emphasize the absence of hysteresis for small fields.

comparison shown in the inset of Fig. 2(a) further confirms that this is a real effect as the background signal [rescaled from Fig. 1(b)] remains a straight line over that field range.

A second observation is the presence of a step in the magnetic moment at low fields (see green arrows) for the no mask sample that accounts for a relatively large fraction of the total signal [see also the zoomed-in view from Fig. 2(d)]. In contrast, as visible from Fig. 2(b) [and corresponding zoomed-in field range from Fig. 2(e)], the step is absent in the mask sample, which exhibits a square loop. Such steps appear in all our no mask TbFe films, independent of composition, whereas the mask samples always show a square hysteresis, consistent with full PMA as previously found for the investigated compositions. ${ }^{8}$ Considering the cut $\mathrm{Tb}_{30} \mathrm{Fe}_{70}$ sample from Fig. 2(f), it is immediately obvious that there is no step in the magnetic moment and that it exhibits a very similar loop to the mask sample from Fig. 2(e) [the magnetic moment is higher due to the fact that the cut piece was larger in size]. Note that, similar to the case of no mask and mask samples from Figs. 2(a) and 2(b), the high magnetic field data (not shown) of the cut sample also reveal an increase in magnetization with respect to the remnant value.

We therefore conclude that the observed steps in the magnetic moment for the no mask sample are not inherent to the TbFe alloy but are an artifact emerging from the coated sample sides. Given that our substrate dimensions are $2.2 \times 2.2 \times 0.275 \mathrm{~mm}^{3}$ [see Fig. 1(a)], the total area of the sides actually amounts to about half of the top area. Therefore, it is not surprising that the material deposited on the sample sides gives rise to a significant signal. Furthermore, the magnetic properties of the sides must be different from those of the top part of the sample (due to sputtering under a different angle compared to the top surface and/or due to the fact that the starting Si substrate is not polished on the sides as it is on the top). To obtain a quantitative measure of the different contributions, we consider the no mask measurement loop as consisting of two different magnetic materials, one soft (e.g., sides signal) and the other hard (e.g., top surface signal), and with moments $\mathrm{m}_{\text {soft }}$ $<\mathrm{m}_{\text {hard }}$. Extracting $\mathrm{m}_{\text {top }}=\mathrm{m}_{\text {hard }}=0.040 \pm 0.004 \mathrm{memu}$ (saturation at $+70 \mathrm{kOe}$ ) from the loop in Fig. 2(a) and considering a mask coverage of $78 \%$ (measured), the expected equivalent moment to the mask sample would be $0.78 \times \mathrm{m}_{\text {top }}=0.031 \pm 0.003 \mathrm{memu}$. This value is in very good agreement with the measured moment [from Fig. 2(b) $] \mathrm{m}_{\text {mask }}=0.032 \pm 0.002 \mathrm{memu}$ for the mask case. The same calculation is performed for our other composition TbFe films yielding results that give similar agreements, although the relative magnitudes of $\mathrm{m}_{\text {soft }}$ and $\mathrm{m}_{\text {hard }}$ are dependent on the film composition.

We also carried out VSM measurements at $100 \mathrm{~K}$ for the three sample configurations of the $\mathrm{Tb}_{30} \mathrm{Fe}_{70}$ film [see Figs. 2(g)-2(i)]. We find that the total magnetic moment is larger compared to the 300 $\mathrm{K}$ case, consistent with previous studies that revealed a strong increase of the magnetic moment at low temperatures. ${ }^{1}$ In addition to the magnetic moment changes, the coercivity is also larger at lower temperatures, as expected. ${ }^{4}$ Similar to the results obtained at $300 \mathrm{~K}$, we only observe the step in the magnetic moment for the no mask case. Notice, however, that the relative contribution of the step magnetic moment to the total moment is lower than at $300 \mathrm{~K}$, so the artifact is also changing with temperature. This is pointing at compositional and/or thickness differences between the top and sides of the (no mask) sample. Consequently, having two different ferrimagnets in the same sample creates difficulties in determining the compensation point of the artifact-free part of the sample (i.e., top), as at that temperature we would still detect the artifact (from the sides). 

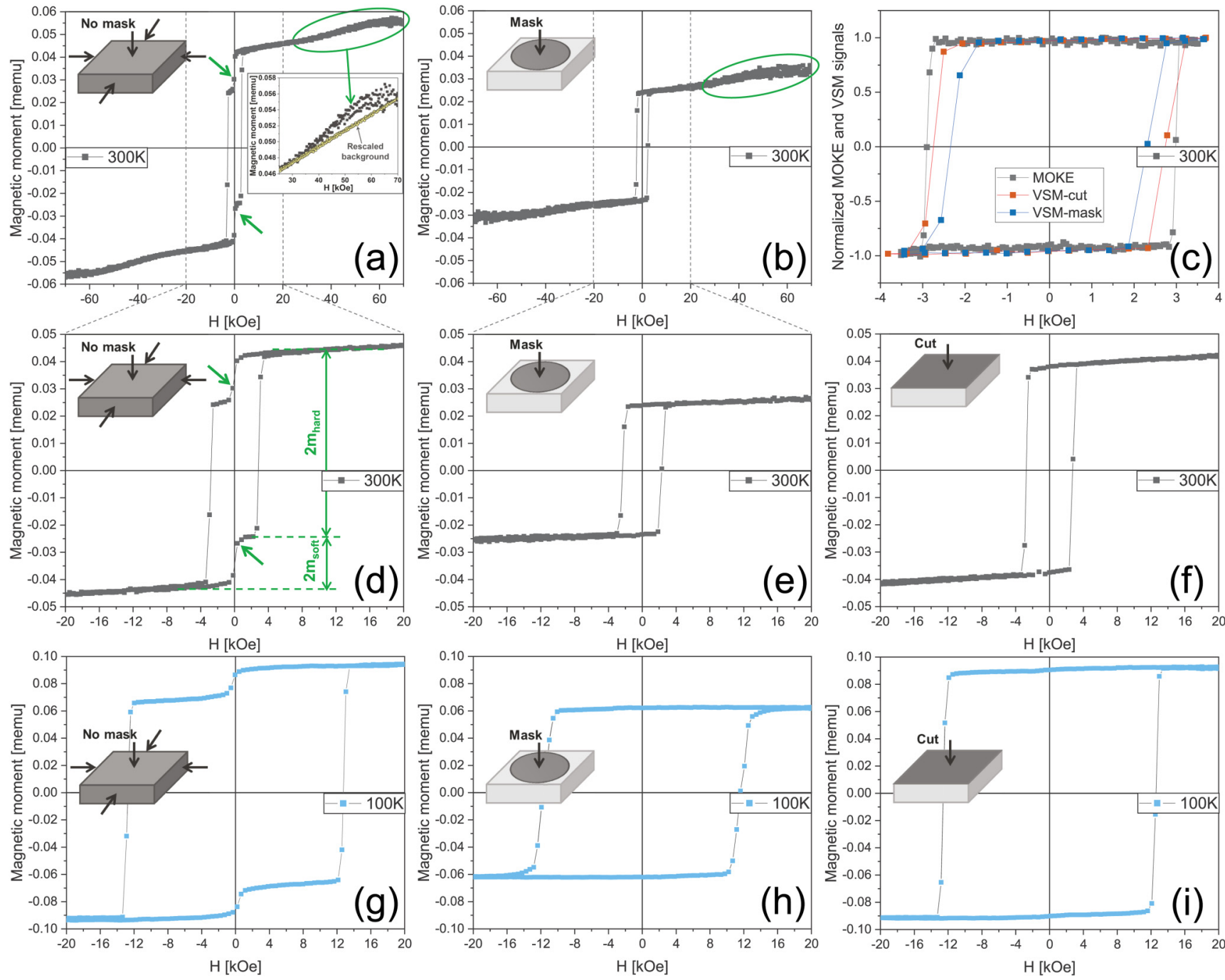

FIG. 2. Out-of-plane $300 \mathrm{~K}$ VSM measurements of a 20 -nm-thick $\mathrm{Tb}_{30} \mathrm{Fe}_{70}$ film for the no mask (a) and mask (b) sample configurations over the entire available field range, with ellipses emphasizing the portion of increased magnetization (see main text for details); the inset in (a) shows a comparison between the $\mathrm{Tb}_{30} \mathrm{Fe}_{70}$ film and background signals between 25 and $70 \mathrm{kOe}$. (d)-(f) Smaller field range out-of-plane $300 \mathrm{~K}$ VSM loops for the no mask, mask, and cut configurations, respectively. (c) $300 \mathrm{~K}$ comparisons of VSM-mask and VSM-cut configurations with the MOKE signal measured on one of the $6 \times 6 \mathrm{~mm}^{2}$ samples. (g)-(i) $100 \mathrm{~K}$ out-of-plane VSM measurements of the same $\mathrm{Tb}_{30} \mathrm{Fe}_{70}$ film for the three sample configurations.

Returning to the cut sample from Fig. 2(f), a closer inspection reveals that it has a coercivity that is about $14 \%$ higher than that of the mask sample. This is more evident from Fig. 2(c), which compares the VSM data acquired on the mask and cut samples to MOKE measurements. Since the coercivities from MOKE and VSM-cut are comparable, we attribute the lower coercivity of the VSM-mask sample to effects caused by the overlaying mask. First, the mask has a finite thickness; second, there is a small gap between the top of the substrate and the mask. Therefore, some shadowing effects near the mask edges (resulting in a reduction of the film thickness and possibly also in a change of the film composition) are to be expected and could facilitate domain nucleation at these locations, thus lowering the film coercivity. Similar trends were observed in our other composition TbFe films, with coercivity changes as high as $20 \%$.

Having established that the step in the magnetic moment is an artifact for the case of TbFe alloys, it is important to assess if sputtering at the substrate sides could affect other systems in a similar way. To that end, the above experiments were also performed for a colloquial RE-free PMA system consisting of [Co/Pt] multilayers, and the results are presented in Fig. 3. Just as in the case of TbFe, we observe the presence of a step (no mask versus mask and cut) and an 

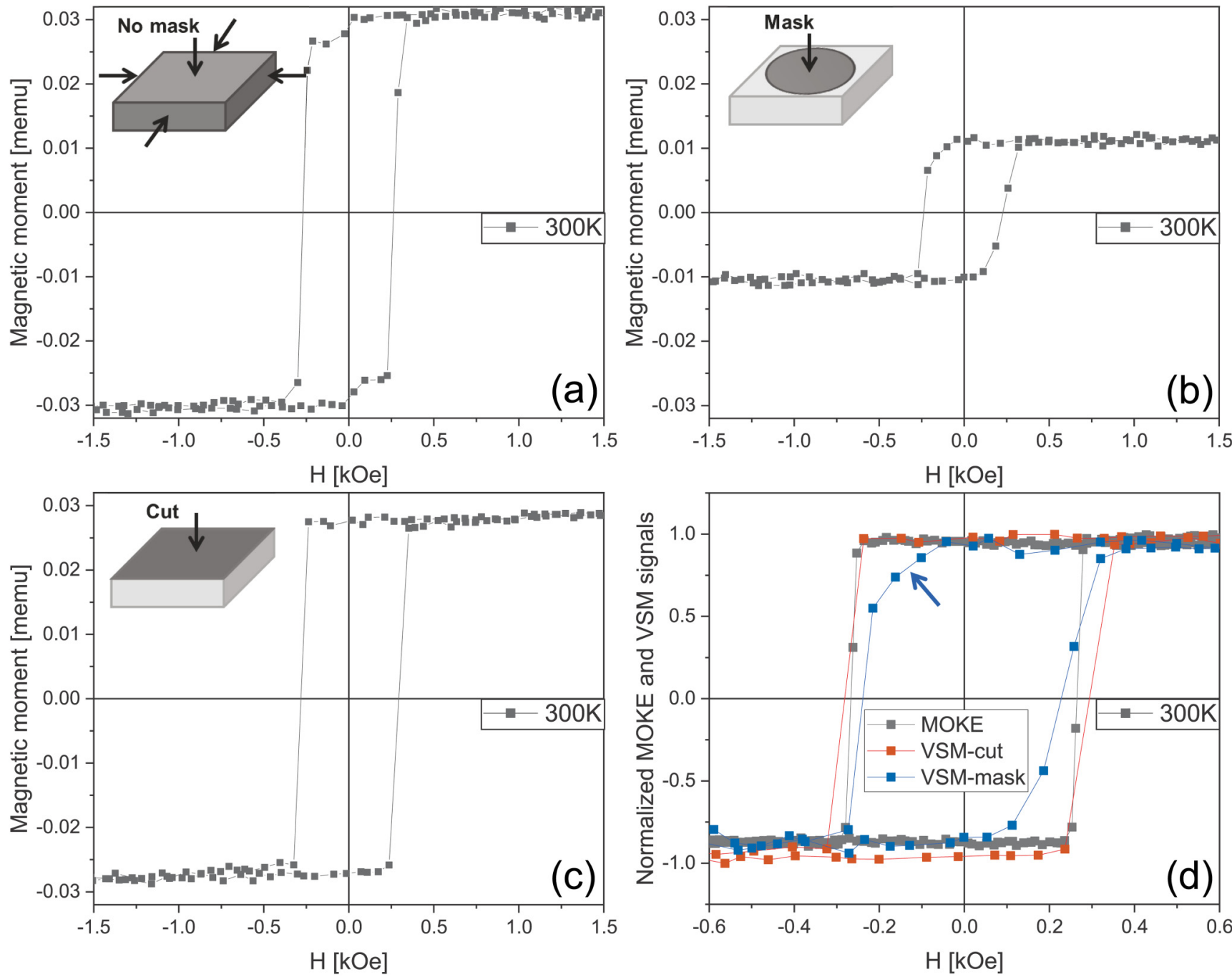

FIG. 3. (a)-(c) Out-of-plane $300 \mathrm{~K}$ VSM measurements of a $\left[\mathrm{Co}_{0.4 \mathrm{~nm}} / \mathrm{Pt}_{0.7 \mathrm{~nm}}\right]_{\times 5}$ multilayer film for three different sample configurations: no mask, mask, and cut, respectively. (d) Comparisons with the MOKE signal measured on one of the $6 \times 6 \mathrm{~mm}^{2}$ samples (the arrow points toward the gradual part of the reversal-see main text).

increase in coercivity by about $20 \%$ (cut versus mask) as also corroborated by MOKE measurements [see comparison graph in Fig. 3(d)]. Also from Fig. 3(d), we observe a more gradual reversal onset for the $[\mathrm{Co} / \mathrm{Pt}]$ film in the mask loop (see arrow) that is absent in the cut and MOKE samples. Even though we do not seek to establish precisely the physical mechanisms behind the observed differences, we point out that the composition gradients and/or thickness inhomogeneities near the mask edges could constitute pinning sites for walls and affect the nucleation field of reversal domains. They can, therefore, have direct bearing on the shape of the reversal curve and/or on the coercivity. We note that these results are independent on the substrate as they also apply to $[\mathrm{Co} / \mathrm{Pt}]$ multilayers grown on thermally oxidized Si substrates (not shown).
Based on the similarities to the MOKE results, one may conclude that the magnetic properties of $\mathrm{TbFe}$ and $[\mathrm{Co} / \mathrm{Pt}]$ are most correctly given by the cut sample configuration. However, we have found that reproducibility can be difficult to achieve consistently based on the cutting practices of each laboratory or even those of different users within the same laboratory. Even more, for some of the cut $[\mathrm{Co} / \mathrm{Pt}]$ films, we observe time-sensitive changes (not shown) that most likely originate from side oxidation and in which the loop shape points to areas that require higher fields to switch. Investigations into the post-cutting oxidation aspect are currently ongoing and will be the topic of a different study. Therefore, we recommend implementing improved masking during growth as a suitable solution for obtaining 
reproducible magnetometry measurements. This can be done by ensuring that the mask is thin compared to the area to be coated and that the gap between the mask and the top of the substrate is supressed by fixing the substrate from the bottom completely against the mask.

Considering the results shown above for the individual systems, investigating the $\mathrm{TbFe} /[\mathrm{Co} / \mathrm{Pt}]_{\times 5}$ exchange-coupled system presents evident complications. Similar steps are also observed in all our no mask $\mathrm{TbFe} /[\mathrm{Co} / \mathrm{Pt}]$ films (not shown), where a superposition of soft features from $\mathrm{TbFe}$ and $[\mathrm{Co} / \mathrm{Pt}]$ leads to complex artifacts that could, in some cases, be confused with characteristics inherent to the desired material system, such as magnetic reversal onsets. As discussed before and already seen in Figs. 2(d) and 2(g), the artifacts are varying with temperature, and as the study of exchange-coupling requires temperature-dependent measurements, they become even more difficult to disentangle from the intrinsic properties of the coupled system.

\section{SUMMARY AND CONCLUSIONS}

In summary, VSM measurements of sputtered RE-TM alloy films and $[\mathrm{Co} / \mathrm{Pt}]$ multilayers reveal artifacts that manifest as soft magnetic components. These artifacts are due to an unwanted material present at the sample sides caused by the way in which the substrate was mounted prior to deposition. The observed steps are not caused by preferential oxidation of the RE element, magnetic impurities, or the presence of very soft grains within our films. By using masking during growth, the sides are not coated and, thus, the appearance of artifacts is suppressed. However, masking causes a decrease in coercivity compared to samples cut post-deposition. As long as one can avoid magnetic contamination from the cutting/handling tools or effects from mechanical deformation, cutting appears to be artifact-free but remains inconvenient for scanning surface probe characterizations and could present timedependent effects due to side oxidation. Therefore, particularly for sputter depositions, where one can grow several samples at once, we suggest to have improved masking that could minimize these problems and then same-batch samples could be used for both magnetometry and surfaces studies. As a final note, the artifacts we find here can be present in many magnetic systems prepared by other common thin-film deposition methods.

\section{ACKNOWLEDGMENTS}

We dedicate this work to the memory of our colleague and friend Miguel A. Marioni. This work was supported by the DACH Project (No. 200021E-160637). The authors thank Claudiu V. Falub for facilitating the MOKE measurements.

\section{REFERENCES}

${ }^{1}$ P. Hansen, C. Clausen, G. Much, M. Rosenkranz, and K. Witter, J. Appl. Phys. 66, 756 (1989).

${ }^{\mathbf{2}}$ V. G. Harris and T. Pokhil, Phys. Rev. Lett. 87, 067207 (2001).

${ }^{3}$ B. Hebler, A. Hassdenteufel, P. Reinhardt, H. Karl, and M. Albrecht, Front. Mater. 3, 1 (2016).

${ }^{4}$ S. Romer, M. A. Marioni, K. Thorwarth, N. R. Joshi, C. E. Corticelli, H. J. Hug, S. Oezer, M. Parlinska-Wojtan, and H. Rohrmann, Appl. Phys. Lett. 101, 222404 (2012).
${ }^{5}$ A. Hassdenteufel, B. Hebler, C. Schubert, A. Liebig, M. Teich, M. Helm, M. Aeschlimann, M. Albrecht, and R. Bratschitsch, Adv. Mater. 25, 3122 (2013). ${ }^{6}$ C. Schubert, A. Hassdenteufel, P. Matthes, J. Schmidt, M. Helm, R. Bratschitsch, and M. Albrecht, Appl. Phys. Lett. 104, 082406 (2014).

${ }^{7}$ B. Hebler, P. Reinhardt, G. L. Katona, O. Hellwig, and M. Albrecht, Phys. Rev. B 95, 104410 (2017).

${ }^{8}$ C. Schubert, B. Hebler, H. Schletter, A. Liebig, M. Daniel, R. Abrudan, F. Radu, and M. Albrecht, Phys. Rev. B 87, 054415 (2013).

${ }^{9}$ M. Tang, Z. Zhang, Y. Zhu, B. Ma, and Q. Y. Jin, Nano-Micro Lett. 6, 359 (2014).

${ }^{{ }^{10}}$ M. H. Tang, Z. Zhang, S. Y. Tian, J. Wang, B. Ma, and Q. Y. Jin, Sci. Rep. 5, 1 (2015).

${ }^{11}$ B. Hebler, S. Böttger, D. Nissen, R. Abrudan, F. Radu, and M. Albrecht, Phys. Rev. B 93, 184483 (2016).

${ }^{12}$ D. Suess and T. Schrefl, Appl. Phys. Lett. 102, 162405 (2013).

${ }^{13}$ V. Skumryev, S. Stoyanov, Y. Zhang, G. Hadjipanayis, D. Givord, and J. Nogués, Nature 423, 850 (2003).

${ }^{14}$ R. F. L. Evans, R. Yánes, O. Mryasov, R. W. Chantrell, and O. Chubykalo-Fesenko, Europhys. Lett. 88, 57004 (2009).

${ }^{15}$ K.-J. Kim et al., Nat. Mater. 16, 1187 (2017).

${ }^{16}$ L. Caretta et al., Nat. Nanotechnol. 13, 1154 (2018).

${ }^{17}$ Y. Hirata et al., Nat. Nanotechnol. 14, 232 (2019).

${ }^{18}$ S. A. Siddiqui, J. Han, J. T. Finley, C. A. Ross, and L. Liu, Phys. Rev. Lett. 121, 057701 (2018).

${ }^{19}$ D. Kim et al., Nat. Mater. 18, 685 (2019).

${ }^{20}$ Y. Shiota, T. Moriyama, and T. Ono, Appl. Phys. Express 12, 083002 (2019).

${ }^{21}$ S.-C. N. Cheng and M. R. Kryder, J. Appl. Phys. 69, 7202 (1991).

${ }^{22}$ H. Basumatary, J. A. Chelvane, D. V. S. Rao, S. V. Kamat, and R. Ranjan, Thin Solid Films 583, 1 (2015).

${ }^{23}$ K. Wang, Y. Huang, Z. Xu, S. Dong, and R. Chen, J. Magn. Magn. Mater. 424, 89 (2017).

${ }^{24}$ K. Wang, S. Dong, Y. Huang, and Y. Qiu, J. Magn. Magn. Mater. 434, 169 (2017).

${ }^{25}$ K. Wang, R. Chen, and Y. Huang, J. Non-Cryst. Solids 450, 82 (2016).

${ }^{26}$ T. R. Gao, X. Y. Xu, S. M. Zhou, J. Li, A.-L. Xia, and B.-S. Han, J. Magn. Magn. Mater. 306, 324 (2006).

${ }^{27}$ N. Casañ-Pastor, P. Gomez-Romero, and L. C. W. Baker, J. Appl. Phys. 69, 5088 (1991).

${ }^{28}$ P. Stamenov and J. M. D. Coey, Rev. Sci. Instrum. 77, 015106 (2006).

29. Hanmin, S. Dongsheng, G. Cunxu, and H. Kim, J. Magn. Magn. Mater. 308, 56 (2007).

${ }^{30}$ R. Höhne, P. Esquinazi, V. Heera, and H. Weishart, Diam. Relat. Mater. 16, 1589 (2007).

${ }^{31}$ A. Bonanni et al., Phys. Rev. B 75, 125210 (2007).

${ }^{32}$ R. Salzer, D. Spemann, P. Esquinazi, R. Höhne, A. Setzer, K. Schindler, H. Schmidt, and T. Butz, J. Magn. Magn. Mater. 317, 53 (2007).

${ }^{33}$ A. Ney, T. Kammermeier, V. Ney, K. Ollefs, and S. Ye, J. Magn. Magn. Mater. 320, 3341 (2008).

${ }^{34}$ P. J. Grace, M. Venkatesan, J. Alaria, J. M. D. Coey, G. Kopnov, and R. Naaman, Adv. Mater. 21, 71 (2009).

${ }^{35}$ M. A. Garcia, E. Fernandez Pinel, J. de la Venta, A. Quesada, V. Bouzas, J. F. Fernández, J. J. Romero, M. S. Martín González, and J. L. Consta-Krämer, J. Appl. Phys. 105, 013925 (2009).

${ }^{36}$ L. M. C. Pereira, J. P. Araújo, M. J. Van Bael, K. Temst, and A. Vantomme, J. Phys. D Appl. Phys. 44, 215001 (2011).

${ }^{37}$ Z. Boekelheide and C. L. Dennis, AIP Adv. 6, 085201 (2016).

${ }^{38}$ L. M. C. Pereira, J. Phys. D Appl. Phys. 50, 393002 (2017).

${ }^{39}$ D. W. Abraham, M. M. Frank, and S. Guha, Appl. Phys. Lett. 87, 252502 (2005).

${ }^{40}$ M. A. Marioni, M. Penedo, M. Baćani, J. Schwenk, and Hans J. Hug, Nano Lett. 18, 2263 (2018).

${ }^{41}$ Keng-Yuan Meng et al., Nano Lett. 19, 3169 (2019).

${ }^{42}$ J. M. D. Coey, J. Appl. Phys. 49, 1646 (1978). 


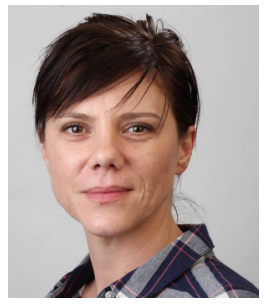

I, Andrada-Oana Mandru, was born in Constanta, Romania, where I grew up and lived until obtaining my undergraduate degree in Technological Physics from Ovidius University in 2008. Before graduation and through some very fortunate interactions with one of my physics professors, I was encouraged to apply for a Master's program in the United States. After taking two English tests (which back then I thought to be most demanding), I was accepted into the M.S. program at the University of Louisiana at Lafayette and later obtained my degree in Applied Physics in 2010. My thesis focused on experimentally determining the elastic constants of magnetostrictive FeGaGe alloys using resonant ultrasound spectroscopy. The two years that I spent at ULL were some of the most difficult, but also a milestone that set my future trajectory. Although English is taught from a young age in Romania, we do not get to practice speaking it very much; as a result, the initial difficulty was to get over my hesitations of speaking and, thus, interacting with people more. Another difficulty was simply being in a totally foreign culture, including academically, which I found very intimidating. While there were many times when I just wanted to pack my bags and return home, I tried to keep reminding myself why I was there and what my goals were. At the same time, I have to admit my curiosity of where this 'adventure' would take me (after all, this was my first time so far away from home). After some time, everything got easier and in addition to becoming friends with some great people, my research interests also got enhanced, I gained more confidence in myself and my capabilities, and therefore decided to pursue a Ph.D. degree.

My pursuits led me to Ohio University, and the six years spent there came with many new experiences, lessons, and surprises. Through meeting and interacting with so many different people from so many different cultures, and also through all the teaching and outreach that I had been exposed to, I learned to be more patient, understanding, and better at managing my time. Through all the research I conducted, the conference attendance and paper writing, I developed more as a researcher; I also learned to always challenge my results, but at the same time to stay realistic and know when to draw the line. On a more personal level and as one of the surprises, at the beginning of the Ph.D., I also met my (now) husband Sean. I graduated from OU with a degree in Physics in 2016. In my dissertation 'Ferromagnetic Thin and Ultra-Thin Film Alloys of Manganese and Iron with Gallium and Their Structural, Electronic and Magnetic Properties', I used mainly a combination of molecular beam epitaxy (as sample growth method) and scanning tunneling microscopy and Auger electron spectroscopy (as surface analysis tools). Encouraged to apply by my Ph.D. advisor, I was selected as one of the finalists and ultimately awarded the 'Russell and Sigurd Varian' Award and the 'Leo M. Falicov' Student Award (in the area of magnetism) at the AVS International Symposium in 2015 based on my dissertation work. After graduation, I also received the '2016 NQPI Outstanding Dissertation' Award in 2017. Earning recognition through these awards showed me that hard work and dedication pay off in the end, but also reinforced the fact that I made the right choice in pursuing such a career (and who does not need reassurance from time to time?).

This brings me to my current position as a Postdoctoral Researcher at Empa-Swiss Federal Laboratories for Materials Science and Technology, in Switzerland. I started this position just before the end of 2016 and my research focuses on magnetron-sputtered magnetic thin-film multilayered systems. I investigate these materials through a combination of vibrating sample magnetometry and high-resolution magnetic force microscopy in UHV, at variable temperature, and in large applied magnetic fields. In addition to performing research, I am also involved in training new students and postdocs, which I enjoy and try to become better at every day. Returning to Europe was a great opportunity to experience research in a different environment and culture, and to continue expanding my previous skill sets while gaining new ones. In addition, Switzerland is a beautiful country with wonderful outdoors, which I very much enjoy discovering.

Having had so many rewarding experiences is what gets me going in the morning and I am very grateful for everything. Looking back at the times before deciding what to do with my life, what I would say to my 16-year-old self, but also to anyone in general regardless of where they come from or what they choose to do, is this: Work hard, persevere, and be dedicated but also be fine with the moments when motivation is at its lowest. Be honest, fair, and speak for yourself. Always try to share your knowledge with others and help them. Try to find a good balance between work and family/personal life. Also remember that you will intersect paths with very different people, some of which will impact you in the most wonderful way and some in the most disappointing way; whatever the case may be, learn from them what to do better or learn what not to do. Last but not least, always remember and value those who helped you get where you are, the ones who ignited your interests, and the ones who gave you inspiration to continue to stay motivated. 